\title{
Associations Between Gambling Games and Gambling Problems: Whole Games Compared with Temporal, Skill Characteristics, and Other Structural Characteristics
}

\author{
Adam S. Goodie ${ }^{1}$ \\ Published online: 31 July 2015 \\ (C) Springer International Publishing AG 2015
}

\begin{abstract}
Research and commentary have been addressed to distinctions regarding problem gambling on the basis of differences in the types of gambling in which individuals engage, such as symptom severity, co-occurring conditions, psychological correlates, and demographic differences. Some progress has been made at the level of the whole game. For example, electronic gaming machine users are more often female and more prone to depressive disorders as well as gambling disorder. Players of other games, such as poker and sports betting, are typically male and prone to substance use disorders. A promising recent trend, however, is to create taxonomies to analyze relevant structural game characteristics that predict gambling problems in a theoretically grounded way. Temporal characteristics such as speed and duration of games have received the most attention, but other relevant characteristics include a skill element, online (Internet) format, and various superficial characteristics. These efforts remain at an early stage, and further development is needed.
\end{abstract}

Keywords Gambling disorder · DSM-5 - Structural characteristics $\cdot$ Gaming $\cdot$ Temporal discounting $\cdot$ Cognitive distortions

This article is part of the Topical Collection on Gambling

Adam S. Goodie

goodie@uga.edu

1 Department of Psychology, University of Georgia, Athens, GA 30602-3013, USA

\section{Introduction}

As legalized gambling expanded in recent decades, the diversity of available forms and formats of gambling also increased, attributable to expanding markets, developing technologies, cultural trends, changes in legal status, and other causes. During the same time period, the seriousness of gambling-related pathologies as a behavioral addiction has become evident [1,2]. For more than 20 years [[3], e.g.,], research and commentary have been addressed to relevant distinctions that may be made regarding problem gambling on the basis of differences in the types of gambling that individuals engage in. Topics of this literature have included (a) differences in severity of gambling symptoms that attend different forms of gambling; (b) cognitive, social, personality, and demographic differences between consumers of different forms of gambling [4], including motivational differences $[5,6]$; and (c) patterns of other comorbid psychiatric conditions that co-occur with use of different forms of gambling, such as a positive association between binge-drinking and skill-based games among college students [7].

\section{Pathology Related to Games}

Whereas some research [4] was addressed to particular facets of electronic gaming machines (EGMs, sometimes known as slot machines or fruit machines), more recent research [8] systematically examined the full range of gambling opportunities, observing several patterns among treatment-seeking problem gamblers, which have since become accepted wisdom. Although women are less likely to gamble and have gambling problems than men overall, they are most heavily represented among EGM users. EGM use was found to be 
associated with elevated levels of gambling symptoms and gambling-related debt, and also with lower levels of treatment seeking. More recently, intriguing neuroscientific research [9] demonstrates that even a single episode of EGM play elicits changes to reinforcement-related brain structures' functioning to respond more to anticipatory cues to winning and less to winning itself. Lotteries and scratch games were also associated not only with lesser gambling expenditures and symptoms but also with relatively low income and high comorbid conditions, such as substance use, abuse and treatment seeking, anxiety, hallucinations, and suicidal ideation and attempt [8]. Gambling on sports was strongly associated with male gender and was additionally associated with higher alcohol and drug use.

Similar findings were reported recently in a cluster analysis reported by Challet-Bouju and colleagues [10••], using a mixed national sample of individuals recruited at gambling venues and through press advertisements. Five classes were identified, consisting predominantly of (1) EGM users; (2) players of deferred lotteries, horse race betting, sports betting, and poker, which are designated as "deferred results" games; (3) roulette gamblers; (4) instant lottery gamblers; and (5) scratch cards gamblers. Classes 1 and 5 gamblers were more frequently female, with class 1 displaying more depressive disorders but less addictive disorders and class 5 displaying less gambling severity but more frequent panic attacks and eating disorders. Classes 2 and 3 were more often male (even more than the sample as a whole, which was $67 \%$ male), with class 2 displaying low harm avoidance and cooperativeness, greater addictive disorders, and some gambling symptoms and class 3 demonstrating higher single-day maximum bets and greater manic or hypomanic episodes. The main distinguishing feature of class 4 was greater history of suicide attempts.

Some systematic differences based on gambling activity have thus been identified in areas of severity, comorbidity, and demographics. Criticism of this approach has emerged, though, posed with special pith in the title of a recent opinion piece by Griffiths and Auer [11••], "The irrelevancy of game-type in the acquisition, development, and maintenance of problem gambling." The thrust of this argument is not that gambling problems are seen identically in the players of all games but that it is structural characteristics of these games, rather than the games in their totalities, that are most fruitfully analyzed to illuminate etiology and treatment approaches to gambling disorder, as well as the basic study of financial risk taking. It is instructive that ChalletBouju et al. united the diverse set of games in class 2 according to the temporal distance between the game and its consequences, as this temporal relation is a critical theme seen in several accounts of relevant game characteristics and one to which we will return.

\section{Structural Characteristics}

In advocating an approach based on structural characteristics rather than whole games, Griffiths and Auer [11••] followed methodological advances in identifying taxonomies of the structural characteristics associated with risk [12]. They found five relatively specific game characteristics as potentially relevant, based on previous research: event frequency, bet frequency, event duration, in-play gambling, and payout interval. These dimensions are motivated by a general impression that "the speed and frequency of the game" (p. 1) are the main force behind the potential for gambling-related harms. The authors point out that the frequency of an "event" (the entity that forms the identity of winning and losing bets, such as the result of a soccer match or the drawing of lottery numbers) may not match the frequency of betting, as one may buy more than one lottery ticket or make multiple distinct bets on a single soccer match. Further, events with relatively long duration may foster "in-play gambling" [13], such as betting on the result of a particular corner kick. Finally, payout interval is used to refer both to the time that elapses from the completion of the event until the payment is made and also the schedule of reinforcement according to which bets are won. As has been noted many times, behaviors reinforced on variable ratio schedules [14], which are characteristic of EGM play, are particularly robust and resistant to extinction.

These characteristics, as a set, leave a number of questions open. Event frequency and bet frequency appear to be usefully distinguished in lotteries, in which one may buy multiple tickets for the same drawing, but not for other games such as poker or sporting event outcomes. For in-game betting, the choice to designate a corner kick as something other than an event may be arbitrary. While it may be reasonable in the case of soccer to designate the higher-order event (the whole soccer game) as the true "event" in distinction to the smaller unit, there are other settings where the designation is less clear. Is the "event" in poker the single hand or the outcome of a sequence of hands across an evening, or a tournament? Also, delay of reinforcement is generally viewed as distinct from the interval associated with a schedule $[15,16]$, such that the "payout interval" characteristic may need further elucidation and, possibly, division into separate concepts.

Interestingly, the earlier research $[10 \bullet \bullet]$, despite a primary focus on games as a whole, did analyze games in terms of two dimensions previously discussed in the literature, namely pure chance versus combined skill and chance [17] and bank games versus social game [18]. The bank versus social dimension reflects that social games have expected value $=0$ because no commercial interest takes a portion of the bets, whereas bank games have expected value $<0$. This pair of potential dimensions is not especially compelling, as the chance-social cell contains only coin tossing and rock-paper-scissors, which are not strongly associated with actual gambling, and the 
combined-social cell contains poker, which is undeniably serious gambling, plus the games of backgammon, bridge, and rummy, which are not. In short, the category of social games serves primarily to separate poker from all other forms of gambling (this may be related to the fact that [17] was addressed solely to poker). Among the bank games, blackjack, sports betting, and horserace betting are considered to contain an element of skill, and a long list of other games (including some poker variants) are considered pure chance games. For these reasons, it is not surprising that the paper's conclusions rested on whole-game considerations rather than specific characteristics.

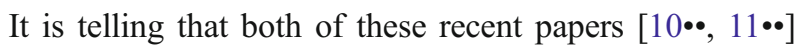
explore potential systems of conceptualizing relevant game characteristics that, in addition to their significant respective limitations, have little overlap when viewed simultaneously (as the sole point of congruence, the concept of event duration appears similar to the designation of deferred gambling assigned to one empirical cluster comprising several diverse games by $\left.\left[10^{\bullet \bullet}\right]\right)$. It is appropriate to conclude that such systems are in the early stages of development.

Rachlin and colleagues [[19••]; updating [20]] provide an example of theoretically grounded models that take account of structural characteristics of games and the literature of temporal discounting. This model assumes that gambling events are experienced and encoded as a string of losses concluding with a single win. Given this model structure, wins are always coded as recent and therefore memorable, and long strings of losses include many which are remote and therefore dim memories. Because the reinforcing value of wins is always recent and strong, and the punishing value of losses is often substantially diminished by the delay from their occurrence to their encoding, gambling in this model is always maintained at greater rates than its normative value would suggest. Assuming further that cumulative gains and losses are calculated following each win, long losing streaks lose their punishing value by the time from the first bet until the eventual win, whereas short losing streaks with favorable resolutions by a quick win are correspondingly advantaged in their reinforcement value. Furthermore, individuals who discount past events more strongly will show this effect more strongly than those with lesser delay discounting. In fact, problem gamblers demonstrate greater delay discounting than nonproblem (but still frequent) gamblers [21].

\section{Skill Characteristics}

A considerable literature has been devoted to the dimension of skill versus luck in various games. Males differentially prefer skill-based games [22], perhaps contributing to their greater rates of gambling problems. EGMs, which are typically associated with high severity levels, are also generally regarded as low-skill games. However, the "luck" and "skill" labels should be applied with care. For example, individual gamblers may incorrectly believe that a low-skill game such as an EGM involves skill, and bet, even excessively, on the basis of this erroneous belief. In this case, it would not be the low actual skill level associated with the game that led the player to (excessively) play it, but rather its high-skill attributes that exist only in the player's belief system.

It is well documented that the illusion of control and other cognitive distortions play important roles in gambling pathology [23, 24••] and its treatment [25]. In particular, the illusion of control and the gambler's fallacy are most prominently demonstrated to predict gambling pathology, with many other distortions having received varying amounts of research authentication with varying methods and varying effect sizes. Therapies that incorporate specifically challenging these cognitive distortions within a broader context of cognitivebehavioral therapy have proven effective.

Further, vagaries in the distinction between luck and skill may not exist solely in the flawed minds of gamblers. Nine out of ten EGM players can correctly detect the difference between a "loose" machine (98\% payback) and a "tight" machine ( $85 \%$ payback) [26]. Thus, even the most prototypically chance-based of betting games has an element of skill: one can realistically expect higher returns by selecting well which machines to play, just as the poker player can expect higher returns by selecting well which hands to play. The analogy has limitations of course. EGMs have a smaller component of skill than poker. But it illustrates that, just as no gambling is based purely on skill, one is hard-pressed to find a game in which skill plays no role. Because the balance between luck and skill is quantitative rather than absolute, the player's perception of the balance may be of critical importance, highlighting the relevance of cognitive factors such as the illusion of control.

\section{Other Characteristics}

Increased attention is being devoted to potential problems associated with online (Internet) gambling [27-30], particularly as it provides a wider array of gaming opportunities than any single brick-and-mortar facility could; it can be more readily engaged without any social contact than most other forms of gambling and is relatively new and thus may reveal unique characteristics yet unknown. One such dimension has been identified: that social (i.e., cost-free) online gaming does lead to significant levels of online gambling [31]. Further, online poker players display greater perception of their own skill, compared with offline gamblers, which is not matched by greater actual skill [32].

Additional research has found superficial features such as sounds, red lights, and social pairs [33] are associated with 
problem gambling behaviors, as the "...casino environment may diminish the time used for reflecting and thinking before acting after losses" (p. 1). Another relevant dimension may be conflict or, in other words, the amount of risk in a gamble, where very high or very low probabilities of winning are considered low conflict due to near-certain advance knowledge of the outcome, whereas moderate-likelihood games are considered high conflict [34].

Comparing among the various classes of structural characteristics, one study [35] shows skill variables more relevant to severity ratings than speed variables. Research on Internetspecific and other characteristics are accelerating in their impact but await more theoretical treatment.

\section{Games or Characteristics: Advantages and Disadvantages}

There is a clear trend in the direction from consideration of games in their entirety to identifying relevant dimensions of those games. This trend appears likely to enhance the productivity of research to discover why, for example, EGMs are associated with greater rates of harm than some other gambling games. It is surely not the name "EGM" that carries this harm but particular features of the game, which will best be elucidated if researchers search for them. It may be revealed that particular features of EGMs, or of poker, lotteries, or any other game, enhance or diminish the risk of harm, which can be leveraged for harm reduction without eliminating the game entirely.

However, it should be recognized that ignoring the game as a whole in research could blind research to important considerations. Games are often associated with distinctive peripheral characteristics, which may not be essential to the game but may contribute to gambling harms. For example, some games are characteristically played in public places with other individuals in relatively close proximity, such as physical EGMs. Bingo is often played in conjunction with charitable purposes and lotteries in support of public goods such as education. Roulette and EGMs are generally played in commercial venues, and sports and poker betting are played robustly both at commercial venues and in private settings within social networks of friends and family [36]. If research pursues only inherent properties of the game, and frequent cultural associates such as typical venue or social impacts are the real cause of harm then the resulting literature may spin its wheels. By the same token, if the cultural associates change, for example, as individuals play virtual EGMs online without others nearby, any changes in harm due to the cultural associates will be concealed by a research focus on properties of the game rather than the game as a whole, including its cultural context.

\section{Conclusions}

There is a clear and mainly positive trend toward focusing on structural game characteristics rather than whole-game identity in investigating differences in how gambling problems including gambling disorder are associated with different available games. The most systematic explorations of this type examine temporal characteristics such as the "speed" of the game, although this designation is need of further refinement. Other promising characteristics include skill components (real or imagined), online presentation, and superficial characteristics. As this research advances and whole-game considerations are predicted to retreat, it is recommended that cultural and other attendant characteristics of whole games not be neglected. Griffiths, Hayer, and Meyer [37] have argued that structural features, both game-based and more distal, as well as games in their totality may have additive impacts on gambling-related harm. Consistent with this, the aim is not to determine which variables are most relevant and to discard others, but to assess and further understand them all.

\section{Compliance with Ethics Guidelines}

Conflict of Interest Adam S. Goodie declares grants and travel accommodations from the National Center for Responsible Gaming unrelated to this article.

Human and Animal Rights and Informed Consent For cited papers on which the author of this article was an author, all studies were approved by the University of Georgia Institutional Review Board and upheld all applicable human rights protections.

\section{References}

Papers of particular interest, published recently, have been highlighted as:

- Of major importance

1. American Psychiatric Association. Diagnostic and statistical manual of mental disorders, (DSM-5 $\left.{ }^{\circledR}\right)$. American Psychiatric Pub. 2013.

2. Clark L, Limbrick-Oldfield EH. Disordered gambling: a behavioral addiction. Curr Opin Neurobiol. 2013;23(4):655-9.

3. Griffiths M. Fruit machine gambling: the importance of structural characteristics. J Gambl Stud. 1993;9(2):101-20.

4. Griffiths MD. The role of cognitive bias and skill in fruit machine gambling. Br J Psychol. 1994;85(3):351-69.

5. Abarbanel BL. Differences in motivational dimensions across gambling frequency, game choice and medium of play in the United Kingdom. Int Gambl Stud. 2014;14(3):472-91.

6. Chantal Y, Vallerand RJ. Skill versus luck: a motivational analysis of gambling involvement. J Gambl Stud. 1996;12(4):407-18.

7. Bhullar N, Simons L, Joshi K, Amoroso K. Relationship among drinking games, binge drinking and gambling activities in college students. J Alcohol Drug Educ. 2012;56(2):58. 
8. Petry NM. A comparison of treatment-seeking pathological gamblers based on preferred gambling activity. Addiction. 2003;98(5): 645-55.

9. Shao R, Read J, Behrens TEJ, Rogers RD. Shifts in reinforcement signalling while playing slot-machines as a function of prior experience and impulsivity. Transl Psychiatry. 2013;3(1):e213.

10.• Challet-Bouju G, Hardouin JB, Renard N, Legauffre C, Valleur M, Magalon D, et al. A gamblers clustering based on their favorite gambling activity. J Gambl Stud. 2014;1-22. The authors present the most important recent clustering of gamblers according to their preferred games. Cluster 1 revealed EGM users who were more female and more prone to depressive disorders. Cluster 2 revealed players of deferred-outcome games who were more male and prone to substance use disorders.

11.• Griffiths MD, Auer M. The irrelevancy of game-type in the acquisition, development, and maintenance of problem gambling. Front Psychol. 2012;3. The authors of this opinion piece laid the conceptual groundwork for considering structural characteristics of games, rather than whole games, in assessing between-game differences in associated patterns of gambling problems, other co-occurring disorders, and demographic characteristics.

12. Meyer G, Fiebig M, Hafeli J, Morsen C. Development of an assessment tool to evaluate the risk potential of different gambling types. Int Gambl Stud. 2011;11(2):221-36.

13. Parke J, Griffiths MD. The role of structural characteristics in gambling. In: Smith G, Hodgins D, Williams R, editors. Research and measurement issues in gambling studies. New York: Elsevier; 2007. p. 211-43.

14. Skinner BF. Science and human behavior. Simon and Schuster; 1953.

15. Mazur JE. Hyperbolic value addition and general models of animal choice. Psychol Rev. 2001;108(1):96.

16. Rachlin H, Green L. Commitment, choice and self-control. J Exp Anal Behav. 1972;17(1):15-22.

17. Bjerg O. Problem gambling in poker: money, rationality and control in a skill-based social game. Int Gambl Stud. 2010;10(3):239-54.

18. Boutin C. Le jeu: chance ou stratégie?: choisir librement la place du jeu dans votre vie. Éditions de l'Homme; 2010.

19.• Rachlin H, Safin V, Arfer KB, Yen M. The attraction of gambling. J Exp Anal Behav. 2015;103(1):260-6. The authors are able to account for several seemingly disparate gambling effects by reconceptualizing the unit of play as any string of losses followed by a win. Applying this behavioral unit to well-established delay discounting models provides a plausible explanation of continuing to gamble despite losses.

20. Rachlin H. Why do people gamble and keep gambling despite heavy losses? Psychol Sci. 1990;1(5):294-7.

21. MacKillop J, Miller JD, Fortune E, Maples J, Lance CE, Campbell WK, et al. Multidimensional examination of impulsivity in relation to disordered gambling. Exp Clin Psychopharmacol. 2014;22:17685.

22. Boldero JM, Bell RC. Chance-and skill-based dimensions underlying young Australians' gambling activities and their relationships with gambling problems and other factors. Int Gambl Stud. 2012;12(2):145-62.

23. Goodie AS. The role of perceived control and overconfidence in pathological gambling. J Gambl Stud. 2005;21(4):481-502.

24.• Goodie AS, Fortune EE. Measuring cognitive distortions in pathological gambling: review and meta-analyses. Psychol Addict Behav. 2013;27(3):730. The authors review the literature of the role of cognitive illusions in gambling disorder. The illusion of control and the gambler's fallacy are most prominently demonstrated to predict gambling pathology, with many other distortions having received varying amounts of research authentication with varying methods and varying effect sizes.

25. Fortune EE, Goodie AS. Cognitive distortions as a component and treatment focus of pathological gambling: a review. Psychol Addict Behav. 2012;26(2):298

26. Dixon MJ, Fugelsang JA, MacLaren VV, Harrigan KA. Gamblers can discriminate 'tight'from 'loose'electronic gambling machines. Int Gambl Stud. 2013;13(1):98-111.

27. Abarbanel BL. Cultural indicators of internet sports betting policy. UNLV Gaming Res Rev J. 2012;16(1).

28. McBride J, Derevensky J. Internet gambling and risk-taking among students: an exploratory study. J Behav Addict. 2012;1(2):50-8.

29. Gainsbury SM, Russell A, Hing N, Wood R, Lubman D, Blaszczynski A. How the Internet is changing gambling: findings from an Australian prevalence survey. J Gambl Stud. 2015;31:115.

30. Dragicevic S, Percy C, Kudic A, Parke J. A descriptive analysis of demographic and behavioral data from Internet gamblers and those who self-exclude from online gambling platforms. J Gambl Stud. 2015;31:105-32.

31. Kim HS, Wohl MJ, Salmon MM, Gupta R, Derevensky J. Do social casino gamers migrate to online gambling? An assessment of migration rate and potential predictors. J Gambl Stud. 2014;1-13.

32. MacKay TL, Bard N, Bowling M, Hodgins DC. Do pokers players know how good they are? Accuracy of poker skill estimation in online and offline players. Comput Hum Behav. 2014;31:419-24.

33. Brevers D, Noël X, Bechara A, Vanavermaete N, Verbanck P, Kornreich C. Effect of casino-related sound, red light and pairs on decision-making during the Iowa gambling task. J Gambl Stud. 2014;1-13.

34. Titz K, Andrus D, Miller J. Hedonistic differences between mechanical game players and table game players: an exploratory investigation on the road to a comprehensive theory for gambling. UNLV Gaming Res Rev J. 2012;6(1):2.

35. Welte JW, Barnes GM, Tidwell M-CO, Hoffman JH. The association of form of gambling with problem gambling among American youth. Psychol Addict Behav. 2009;23:105-12.

36. Meisel MK, Clifton AD, MacKillop J, Miller JD, Campbell WK, Goodie AS. Egocentric social network analysis of pathological gambling. Addiction. 2013;108:584-91.

37. Griffiths M, Hayer T, Meyer G. Problem gambling: a European perspective. Problem gambling in Europe: challenges, prevention, and interventions. 2009. 